\title{
Evaluation of an At-Home-Use Prostate Massage Device for Men with Lower Urinary Tract Symptoms
}

\author{
Jillian L. Capodice, Brian A. Stone and Aaron E. Katz
}

Department of Urology, Columbia University Medical Center, NY, USA

\begin{abstract}
Introduction: Treatment of lower urinary tract symptoms (LUTS) in men due to benign prostatic hyperplasia (BPH) or chronic prostatitis/chronic pelvic pain syndrome (CP/CPPS) are difficult for both the patient and the clinician. In office prostatic massage has been documented to provide symptom relief but use of at-home massage has not been determined. We performed a retrospective analysis of data from men utilizing an at-home-use prostate device to examine disease, treatment characteristics, and symptom relief in order to ascertain whether evidence exists to perform a clinical trial.

Methods: Data on 154 consecutive men was reviewed and subjects evaluated in two Groups, BPH w/ LUTS (Group 1) and CP/CPPS w/ LUTS (Group 2). All subjects completed the National Institutes of Health, Chronic Prostatitis Symptom Index (CPSI) at baseline and follow-up. Demographics, scores on the CPSI, duration of device use, self-reported symptom assessment, and comments were analyzed.

Results: Of 154 men, 115 were analyzed. Of the 115, 90 (78.3\%) were in Group 1 (BPH w/LUTS) and 25 (21.7\%) were in Group 2 (CP/CPPS w/LUTS). The average age was 64.48 years \pm 10.86 (average \pm SD) vs $46.68 \pm 12.5$ (Group $1-2$, respectively). In Group 1 total CPSI score from baseline to follow-up were $(11.61 \pm 7.07$ (mean $\pm \mathrm{SD})-6.63 \pm 5.20, \mathrm{p}=$ $0.0001)$. In Group 2 total scores from baseline to follow-up were $(16.67 \pm 7.0 v s 11.48 \pm 5.84(5.20, \mathrm{p}=0.0127)$. Other self-reported comments included 16/115 (13.9\%) of subjects unsure about proper use/application and 10/115 (8.6\%) reporting rectal soreness.

Conclusions: The preliminary findings suggest that a clinical trial of a novel at-home-use prostatic massage device is warranted.
\end{abstract}

Keywords: Prostate device, non-conventional treatment, LUTS, BPH, CP/CPPS.

\section{INTRODUCTION}

Treatment of bothersome lower urinary tract symptoms (LUTS) in men may be related to a number of conditions such as benign prostatic hyperplasia (BPH), chronic prostatitis/chronic pelvic pain syndrome (CP/CPPS), or bladder conditions such as interstitial cystitis or overactive bladder [1-3]. Regardless of etiology, male LUTS remains a challenge for both the clinician and the patient. For prostate related conditions, $\mathrm{BPH}$ and more frequently, $\mathrm{CP} / \mathrm{CPPS}$, the utility of in-office prostatic massage for symptom relief has been documented with varying results $[4,5]$. However, continued treatment via subsequent office visits or the potential utility of performing massage at home has not been evaluated. A novel prostatic massage device which enables men with LUTS to perform at home prostatic massage has been recently manufactured. However the mechanism for prostate massage still needs to be characterized and "dosage" with regard to frequency and duration needs to be investigated regardless of whether the procedure is done either in the office or at home. We performed a retrospective analysis of data from men utilizing an at-home-use prostate

*Address correspondence to this author at the Department of Urology, Columbia University Medical Center, 161 Fort Washington Avenue, Department of Urology, $11^{\text {th }}$ Floor, New York, NY 10032, USA; Tel: 212305-6408; Fax: 212-305-0106; E-mail: aek4@columbia.edu device in order to examine disease and treatment characteristics and how they correlated with patient satisfaction and symptom relief in order to ascertain whether evidence exists to warrant a clinical trial where the safety and efficacy of this novel device could be tested for men with LUTS.

\section{METHODS}

Data on 154 consecutive men that reported using an athome prostate massage device from July 2006-July 2008 was reviewed. The device [Pro-State ${ }^{\circledR}$, High Island Health, LLC, Houston, TX] is a medical-grade acetal-based device that was designed to emulate an in-office digital prostate massage. The device allows for trans-rectal prostate stimulation and perineal stimulation based on the location of a traditional acupuncture point, CV1 (located in the center of the perineum), as well as contraction of the pubococcygeus (PC) muscle in order to strengthen muscles of the male pelvic floor (Figs. 1A, 1B). The subjects were evaluated based on two Groups; Group 1 consisted of men diagnosed with BPH and LUTS symptoms and Group 2 consisted of men diagnosed with CP/CPPS (Types IIIA and B) and LUTS symptoms. All subjects must have completed the National Institutes of Health, Chronic Prostatitis Symptom Index (CPSI) at baseline and follow-up period (range $<4$ weeks to $>24$ weeks). Characteristics that were analyzed included demographics, scores on the CPSI (total, pain, urinary, 
quality of life, symptom (pain + urinary), duration of device use, self reported symptom assessment based on a 1-5 rating scale, and additional self reported comments. We excluded patients who had a history of prostate, colo-rectal or other cancers, active upper or lower urinary tract infection, acute or chronic bacterial prostatitis, primary erectile dysfunction, and history of kidney stones.

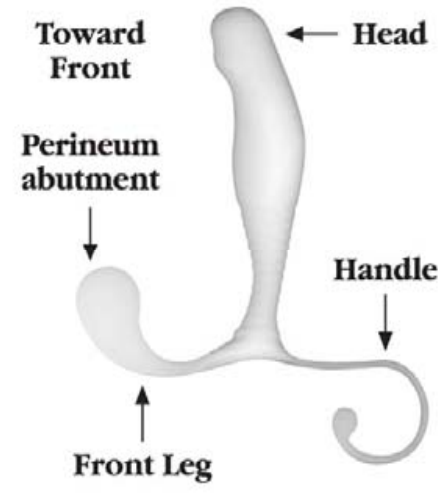

Fig. (1A). Side view of the prostate massage device labeled to indicate proper placement in to the rectum.

\section{RESULTS}

Of 154 men that reported using the device 115 were analyzed. Of the 115 men, $90(78.3 \%)$ had BPH and 25 $(21.7 \%)$ were in the CP/CPPS Group. The average age of the BPH Group was 64.48 years \pm 10.86 (average \pm SD) and $46.68 \pm 12.55$ (average $\pm \mathrm{SD}$ ) for the CP/CPPS Group respectively (Table 1). The duration of device use ranged from $<4$ weeks to $>24$ weeks and the percentage of use in each Group was as follows: $>4$ weeks $(63.6$ vs 76) $(\% \mathrm{BPH}$ vs CP/CPPS), 4-12 weeks (22.2 vs 20), 12-24 weeks (14.4 vs $4)$ and $>24$ weeks (0). There was no statistical difference in duration of use between the two Groups (Table 1). Pain, urinary function, quality of life, symptom (pain + urinary), and total scores on the CPSI were analyzed within each Group and then between Groups. In the BPH Group, differences on the total CPSI score from baseline to followup were $(11.61 \pm 7.07$ (mean $\pm \mathrm{SD})$ vs $6.63 \pm 5.20, \mathrm{p}=$ $0.0001)$. In the $\mathrm{CP} / \mathrm{CPPS}$ total scores from baseline to follow-up were $(16.67 \pm 7.0$ vs $11.48 \pm 5.84$ (5.20, p = $0.0127)$. Individual subset scores are shown in Table 2. When comparing baseline and follow-up scores in $\mathrm{BPH}$ versus $\mathrm{CP} / \mathrm{CPPS}$ subjects, we found that $\mathrm{CP} / \mathrm{CPPS}$ subjects had a higher total score at baseline $v s \mathrm{BPH}$ subjects $(16.67 \pm$

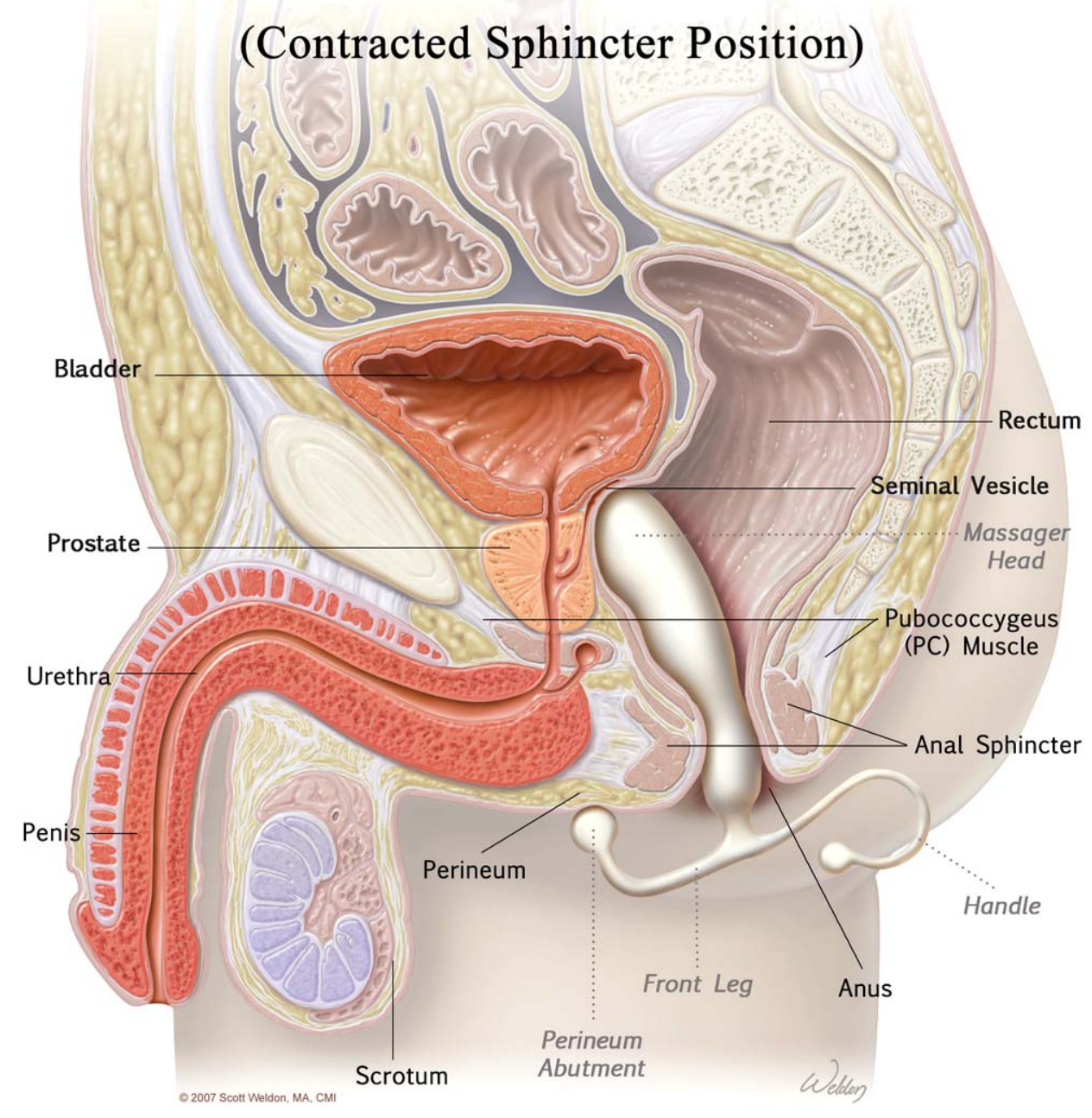

Fig. (1B). Anatomical rendering (sagittal view) of the prostate massage device in place within the rectum. 
Table 1. Demographics

\begin{tabular}{|l|c|c|c|}
\hline \multicolumn{1}{|c|}{ Characteristic } & BPH with LUTS & CP/CPPS with LUTS & P Value \\
\hline \hline No. evaluable (\%) & $90(78.3)$ & $25(21.7)$ & N/A \\
\hline Age (average \pm SD) & $64.48 \pm 10.86$ & $46.68 \pm 12.55$ & N/A \\
\hline Race: No (\%) & $1(1.1)$ & $3(12)$ & N/A \\
Asian & $1(1.1)$ & $1(4)$ & \\
Black & $88(97.8)$ & $21(84)$ & \\
White & $57(63.3)$ & $19(76)$ & \\
\hline Duration of device use: No (\%) & $20(22.2)$ & $5(20)$ & 0.25 \\
$<4$ weeks & $13(14.4)$ & $1(4)$ & 0.74 \\
4-12 weeks & $0(0)$ & $0(0)$ & 0.087 \\
12-24 weeks & & & - \\
$>24$ weeks & & & \\
\hline
\end{tabular}

Table 2. Mean (SD) Summary Statistics of the CPSI in Subjects with LUTS

\begin{tabular}{|c|c|c|c|c|c|c|}
\hline NIH-CPSI & BPH Baseline & BPH Follow-Up & BPH P Value & CP/CPPS Baseline & CP/CPPS Follow-Up & CP/CPPS P Value \\
\hline \hline Pain Scores & $3.09(3.40)$ & $1.55(2.34)$ & $0.008^{*}$ & $5.38(3.84)$ & $3.84(2.91)$ & 0.129 \\
\hline Urinary score & $3.71(2.68)$ & $2.2(2.23)$ & $0.006^{*}$ & $4.71(3.02)$ & $3.90(2.55)$ & 0.3533 \\
\hline QOL score & $4.81(2.62)$ & $2.87(2.24)$ & $0.001^{*}$ & $6.57(2.73)$ & $5.05(1.75)$ & 0.373 \\
\hline $\begin{array}{c}\text { Symptom score } \\
=\text { Pain + Urinary }\end{array}$ & $6.80(4.95)$ & $3.76(3.74)$ & $0.0001^{*}$ & $10.10(4.96)$ & $6.43(4.75)$ & $0.0189^{*}$ \\
\hline Total Score & $11.61(7.07)$ & $6.63(5.20)$ & $0.0001^{*}$ & $16.67(7.0)$ & $11.48(5.84)$ & $0.0127^{*}$ \\
\hline
\end{tabular}

CPSI = National institutes of health-Chronic prostatitis symptom index.

$*=\mathrm{p}<0.05$ : unpaired t-test.

7.0 vs $11.61 \pm 7.07, \mathrm{CP} / \mathrm{CPPS}$ vs $\mathrm{BPH}$, respectively), as well as higher scores in all the CPSI domains (Table 2). Selfreported symptom assessment was rated on a 1-5 scale: $1=$ has not helped symptoms, $2=$ slight improvement, $3=$ moderate to good improvement, $4=$ very good improvement, $5=$ completely alleviated symptoms (Table 3). Overall 7.7 and $12 \%(\mathrm{BPH}$ and $\mathrm{CP} / \mathrm{CPPS}$, respectively) of subjects reported that the device did not help their symptoms and 46.7 and $40 \%(\mathrm{BPH}$ and $\mathrm{CP} / \mathrm{CPPS}$, respectively) reported very good symptom improvement. Of other self-reported comments included, 16/115 (13.9\%) of subjects were unsure about proper use/application, 10/115 (8.6\%) reported rectal soreness, and $1 / 115(0.87 \%)$ reported rectal bleeding. Positive reported comments included 10/115 (8.6\%) that experienced increased sexual function and ejaculate (Table 4).

Table 3. Self Reported Symptom Assessment

\begin{tabular}{|l|c|c|}
\hline \multicolumn{1}{|c|}{ Description } & BPH & CP/CPPS \\
\hline \hline No (\%) & & \\
1 = has not helped & $7(7.7)$ & $3(12)$ \\
2 = slight improvement & $11(12.2)$ & $7(28)$ \\
3 = moderate to good improvement & $26(28.8)$ & $2(8)$ \\
$4=$ very good improvement & $42(46.7)$ & $10(40)$ \\
$5=$ completely alleviated symptoms & $4(4.4)$ & $3(12)$ \\
\hline
\end{tabular}

Table 4. Self Reported Comments

\begin{tabular}{|l|c|}
\hline \multicolumn{1}{|c|}{ Description } & No (\%) \\
\hline \hline Increased sexual function and ejaculate & $10(8.6)$ \\
Rectal soreness/uncomfortable & $10(8.6)$ \\
Rectal bleeding & $1(0.87)$ \\
Unsure about proper use/application & $16(13.9)$ \\
\hline
\end{tabular}

\section{DISCUSSION}

Lower urinary tract symptoms (LUTS) which include voiding and pelvic symptoms are common in men with benign prostatic hyperplasia (BPH) and chronic prostatitits/ chronic pelvic pain (CP/CPPS) [1-3]. The use of prostate massage for the detection of bacteria and further analysis of seminal and post-massage voided urine has been previously described $[4,5]$. Utility of in-office prostate massage has also been documented as a procedure that may alleviate LUTS symptoms $[4,6]$. Given the need to alleviate LUTS symptoms in men with $\mathrm{BPH}$ or $\mathrm{CP} / \mathrm{CPPS}$, who we retrospectively evaluated data on men elected to use an athome-use prostate massage device to reduce their symptoms in order to determine if there was evidence to warrant a clinical trial that may help to determine safety, efficacy, dosage and frequency of use.

Our results demonstrate that a number of men were using the device. The average age of BPH subjects and CP/CPPS subjects was consistent with known disease characteristics. In general, we found that the majority of subjects were using the device for a short period of time ( $<4$ weeks) prior to completing the follow-up questionnaire but there were 
significant reductions in the total CPSI scores in both Groups. Interestingly, the pain and urinary sub scores of $\mathrm{CP} / \mathrm{CPPS}$ subjects were not statistically different at baseline versus follow-up, but all domain scores of the BPH subjects were statistically significant. Moreover, about half of the subjects reported very good improvement. Two reported concerns of men were that the device was difficult to use and the men were unsure of proper application, frequency, and duration of use. This device may have various advantages over traditional in-office prostate massage including the potential for increased frequency, which may in turn alleviate symptoms more rapidly, and less stigma or mental burden on the patient when using it in the privacy of the home. In addition, spanning a decade with over 250,000 devices in use, no serious adverse events have been reported, providing preliminary evidence about the safety of its use. However, further studies of merit should include clinical research involving evaluation of safety, testing of optimal duration, and frequency of use.

There are several limitations to the present evaluation. First, it is a retrospective review and there is no direct comparison between the device and traditional in-office prostate massage. While we attempted to minimize the bias of a retrospective study by collecting objective data and utilizing a validated questionnaire, it can be argued that utilization of the CPSI as a measurement tool in men with $\mathrm{BPH}$ is not valid. However, the reason we utilized the CPSI as a measurement tool for men with LUTS symptoms related to $\mathrm{BPH}$ or $\mathrm{CP} / \mathrm{CPPS}$ was because we wanted to capture reported pain in the BPH Group that is not commonly captured by quantitative questionnaires usually used in $\mathrm{BPH}$ patients such as the AUA and IPSS symptom scores, both of which primarily focus on voiding symptoms. Another limitation is that more data on characteristics of use were not measured; for example, the duration of device use was recorded but the frequency of use (i.e., number of times used per week) was not standardized. Additionally, while it was reported that many of these men were refractory to conventional pharmacologic measures, the dosage and type of medications (i.e., alpha-blockers, non-steroidal antiinflammatory agents, antibiotics, etc.) were not recorded. Finally, the demographics of this population are relatively homogenous, and more data needs to be collected on race and anthropomorphic characteristics as the device is available in three sizes based on the height of the user.

In conclusion, traditional in-office prostate massage has demonstrated potential benefit in alleviating LUTS symptoms in men with BPH and CP/CPPS. These significant results demonstrate that the application of an at-home-use prostate massage device may relieve LUTS in men with $\mathrm{BPH}$ and CP/CPPS. However, dosage with regard to frequency and duration need to be determined and monitoring of safety must be considered. Based on these findings we conclude that the at-home-use prostate massage device warrants further analysis in a controlled prospective clinical trial.

\section{DISCLOSURES}

Jillian L. Capodice has provided consulting advice to High Island Health, LLC.

\section{REFERENCES}

[1] Nickel JC, Barkin J, Koch C, Dupont C, Elhilali M. Finasteride monotherapy maintains stable lower urinary tract symptoms in men with benign prostatic hyperplasia following cessation of alpha blockers. Can Urol Assoc J 2008; 2(1): 16-21.

[2] Nickel JC. Role of alpha1-blockers in chronic prostatitis syndromes. BJU Int 2008; 101(Suppl 3): 11-6.

[3] Andersson KE. LUTS treatment: future treatment options. Neurourol Urodyn 2007; 26(6 Suppl): 934-47.

[4] Mishra VC, Browne J, Emberton M. Role of repeated prostatic massage in chronic prostatitis: a systematic review of the literature. Urology 2008; 72(4): 731-5.

[5] Nickel JC, Alexander R, Anderson R, et al. Prostatitis unplugged? Prostatic massage revisited. Tech Urol 1999; 5(1): 1-7.

[6] Nickel JC, Downey J, Feliciano AE Jr, Hennenfent B. Repetitive prostatic massage therapy for chronic refractory prostatitis: the Philippine experience. Tech Urol 1999; 5(3): 146-51. 